\title{
Optimization of Duty Ratio of Metallic Grating Arrays for Infrared Photodetectors
}

\author{
Dong Liu ${ }^{1}$, Yongqi Fu, ${ }^{1, *}$ Lechen Yang ${ }^{1}$, Baoshun Zhang ${ }^{2}$, Haijun $\mathrm{Li}^{2}$, Kai Fu ${ }^{2}$, Min Xiong ${ }^{2}$ \\ ${ }^{1}$ School of Physical Electronics, University of Electronic Science and Technology of China, \\ Chengdu, China \\ ${ }^{2}$ Suzhou Institute of Nano-Technology and Nano-Bionics, CAS, Suzhou, China \\ E-mail: 'yqfu@uestc.edu.cn \\ Received May 27, 2011; revised June 25, 2011; accepted July 6, 2011
}

\begin{abstract}
Influence of duty ratio of metallic gratings applied in quantum well infrared photodetector (QWIP) with detection ranging from $3 \mu \mathrm{m}$ to $5 \mu \mathrm{m}$ was studied in this paper. The influence on longer enhanced wavelength working at infrared waveband was investigated. A relationship between the duty ratio and the enhanced peak intensity is given. Some results can be applied to optimize the enhanced efficiency of the metallic gratings.
\end{abstract}

Keywords: Metallic Gratings, Duty Ratio, Infrared Waveband, QWIP

\section{Introduction}

Recently, many research papers report the enhanced and localized surface plasmon due to the unique feature of extraordinary transmission [1-5]. As well known, to excite surface plasmon, approaches of both prism and grating coupling are applicable. The former needs an appropriate angle to form perfect total reflection which depends on the thickness of the metal film [6,7]. However, period and duty ratio of a metallic grating directly influence the position of the enhanced resonant peak and corresponding values. As a coupling layer of quantum well infrared photodetector (QWIP), it is necessary to consider the feasibility while manufacture QWIP distributed as arrays in large-area [8]. Therefore, the grating coupling will be more appropriate for the infrared imaging devices because of its smaller volume and good controllability in comparison to the approach of prim coupling. To utilize the enhancement of surface plasmon (SP), we investigated the characteristics and fundamental issues of the metallic gratings in the view of optimization of the duty ratio. Investigation of the optimized duty ratio was carried out for the purpose of improving enhanced resonant peak in the infrared waveband.

\section{Theory Background and Computational Setup}

For calculation of the metallic grating, there are some important parameter settings to be considered firstly. The type of the gratings, the period of the arrays, the arrangement mode and size of the holes are all the crucial factors [9-12]. Some papers reported the calculation of the array period by means of the formulas of dispersion relations as follows $[1,7,13,14]$ :

$$
\begin{aligned}
& \lambda_{S P}(i, j)=\frac{a_{0}}{\sqrt{i^{2}+j^{2}}}\left(\frac{\varepsilon_{1} \varepsilon_{2}}{\varepsilon_{1}+\varepsilon_{2}}\right)^{1 / 2} \\
& \lambda_{S P}(i, j)=\frac{\sqrt{3} a_{0}}{2 \sqrt{i^{2}+j^{2}-i j}}\left(\frac{\varepsilon_{1} \varepsilon_{2}}{\varepsilon_{1}+\varepsilon_{2}}\right)^{1 / 2}
\end{aligned}
$$

where, $a_{0}$ is the period of the grating, $\varepsilon_{1}$ and $\varepsilon_{2}$ is the dielectric constants of the medium and metal respectively, and $i$ and $j$ are the integral numbers. Equation (1) is for the array with square cross section. Equation (2) is the array with regular triangle cross section which is calculated by the similar method of phase-matching. The momentum of SP matches the momentum of regular triangle crystal lattice the incident photon. However, the radius of the hole, the thickness of metal film and the hole array will be the three important factors to be determined. Here, we choose the metallic gratings consist of Au film with characteristics of stability and the hollow holes embedded in the sapphire substrate. Though the surface plasmon is sensitive to thickness, the point of this paper is about the duty ratio of the metal gratings. Hence, the $\mathrm{Au}$ film was set as $50 \mathrm{~nm}$, which is an optimized thickness 
as [10]. Shape of the periodic arrange of air holes is regular triangle. We chose a regular triangle lattice instead of square lattice because the former has the wide bandwidth of enhancement as well as large maximal transmission domain due to its symmetrical geometry, and thus the Equation (2) will be practical in the numerical calculation [12]. The simulation model in the software FDTD solutions was established, as shown in Figure 1.

The computational numerical calculation was carried out for the purpose of deriving optimized duty ratio for the gratings in infrared waveband and focus on the midinfrared as well as far infrared where the photodetector works. Therefore, we chose the points in the range from $5 \mu \mathrm{m}$ to $9 \mu \mathrm{m}$ and calculated the period for each corresponding enhanced peak firstly. Then we can obtain the enhanced peak positions and corresponding array periods, as shown in Table 1. For each corresponding periods, we chose a series of radius which match along with the duty ratio as $0.04,0.06,0.08,0.10,0.12,0.14,0.16,0.18,0.20$, $0.25,0.30,0.35,0.40,0.45$, and 0.50 , respectively. Calculation of the transmission spectrum was performed by means of finite difference and time domain (FDTD) algorithm [15].

By calculation, we obtained seven groups of data, as shown in Figure 2. The $\mathrm{x}$-axis denotes the duty ratio of the metallic grating, and the $y$-axis represents the enhanced peak value for each duty ratio. With increasing of the period of the array, the enhanced peak value decreases and the optimizing duty ratio increases accordingly. As can be seen in Figure 2, we picked up the data with the relationship between array period and the enhanced peak intensity, and re-plotted, as shown in Figure 3. It is a quadratic power curve with nearly linear

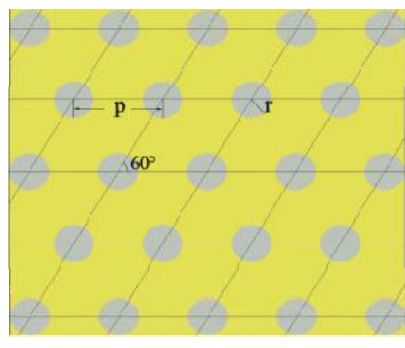

(a)

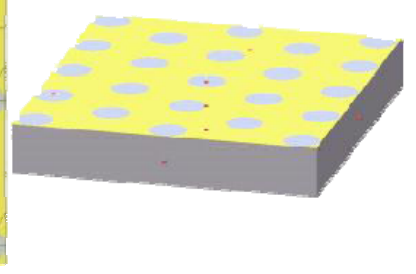

(b)
Figure 1. (a) Vertical view of the model, $p$ is the period of the array, $r$ is the radius of the holes; (b) the perspective view of the model and the material from the bottom to the top is sapphire and Au film respectively.

Table 1. Enhanced peaks and corresponding array periods.

\begin{tabular}{cccccccc}
\hline Enhanced Peak $(\mu \mathrm{m})$ & 5 & 6 & 7 & 7.5 & 8 & 9 & 10 \\
Period $(\mu \mathrm{m})$ & 3.4 & 4.2 & 5.4 & 6.1 & 6.7 & 8.5 & 10.7 \\
\hline
\end{tabular}

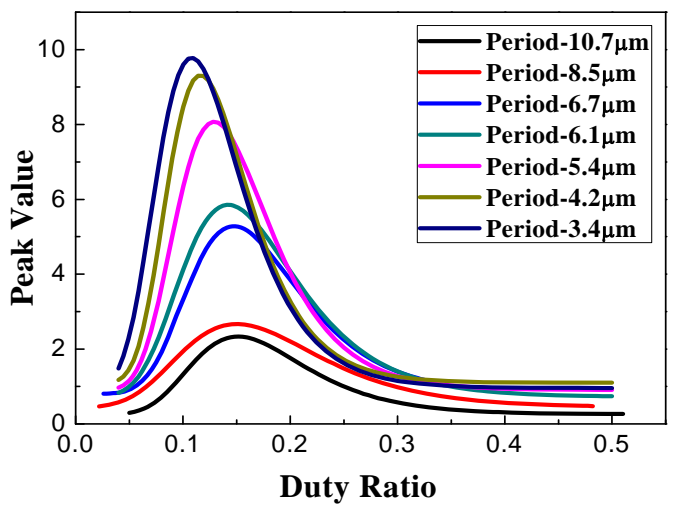

Figure 2. Duty ratio vs. peak values for different period arrays. The duty ratio is ranging from 0.04 to 0.50 and calculated the peak value.

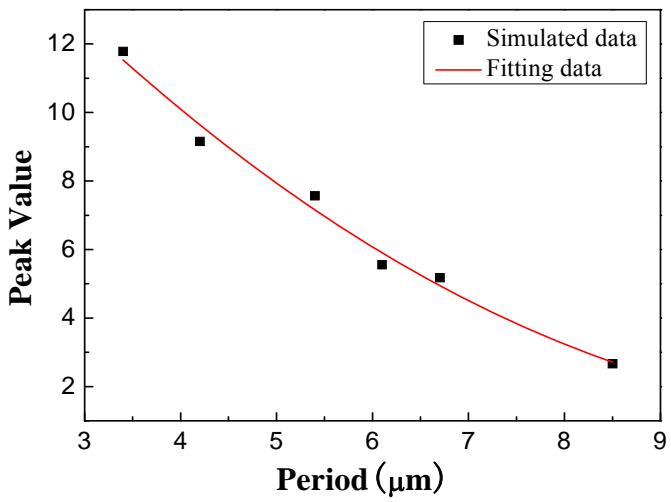

Figure 3. Period of array vs. its corresponding enhanced peak intensity.

relationship. With increasing of the array period, the intensity decreases. This is a qualitative analysis and suggests a rough changing tendency. The intensity is calculated assuming incident beam intensity with value of 1 . However, the most important point of our work is searching the relationship between enhanced wavelength and the duty ratio which can be a reference in the designing of the metallic gratings. As can be seen from Table 1 and Figure 2, for each period it corresponds to an enhanced wavelength and an optimizing duty ratio which indicates the duty ratio when the y-axis value approaches to be maximum for each curve, as shown in Figure 2. Hence, it is easy to obtain the relationship of enhanced wavelength and the optimizing duty ratio. Figure 4 shows the relationship. And the fitting formula is shown as follows:

$$
y=0.14308-0.02088 x+0.00265 x^{2}
$$

Generally speaking, the enhancement and locality of the two-dimensional (2D) metallic gratings are always crucial issues because they play an important role in the design. Therefore, it requires ensuring its enhanced peak 


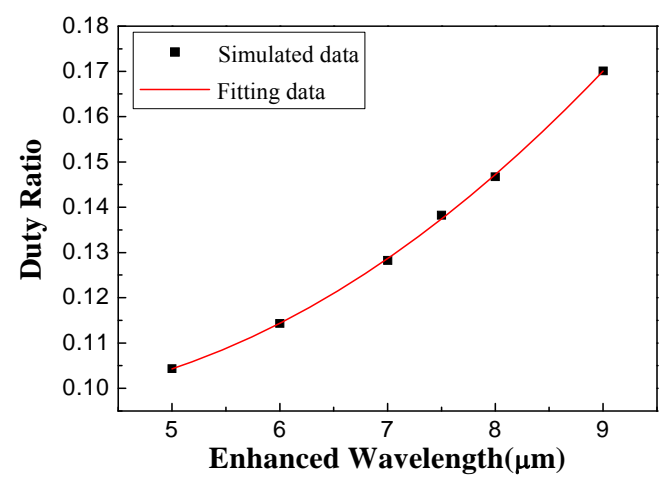

Figure 4. Every enhanced wavelength and its corresponding optimizing duty ratio.

wavelength exactly.

We can find its optimizing duty ratio from this curve once the response wavelength is determined. Figure 4 gives us directly the guideline in the design. Corresponding to each response wavelength we can derive the optimized duty ratio for a strongest enhanced peak value.

\section{Results and Discussion}

As can be seen, the effect of surface plasmon becomes less effective while the enhanced peak intensity drops being weak [16]. However, it can be seen from Figure 3 that certain period value is minimum which will cause the surface plasmon losing its effectiveness of enhancement obviously when the period increases. The duty ratio of the most enhanced peak increases in this case. When the period is large enough, the duty ratio closes to the case that there is a few holes only on the Au film. The peak intensity will equal to that of the light source intensity [17-19]. For different sizes of array structures, it needs different duty ratios so as to obtain the optimized enhancement which is in the same level of orders of magnitude of the enhanced wavelength $[20,21]$. Similarly, the changing rule is a nonlinear curve, when the curve close to a value which requires duty ratio turns to be infinite. Then the surface plasmon enhancement loses its effectiveness [21-24]. This result proves further that surface plasmon effect dose not fit for jumbo size structure. To validate whether this changing rules is exact or not, we collected more results to check it. By choosing any enhanced wavelength, such as $4.5 \mu \mathrm{m}$, then calculate the array period using Equation (2), and the wavelength is almost $2.918 \mu \mathrm{m}$. The corresponding optimized duty ratio for such a responding wavelength will be close to 0.1028 . And the radius can be calculated to be 0.4912 $\mu \mathrm{m}$. This is an analysis result from the curve in Figure 4 theoretically, whereas change its radius in the simulations. We calculated the results as shown in Figure 5. It can be seen that assumes a certain radius which can gen-

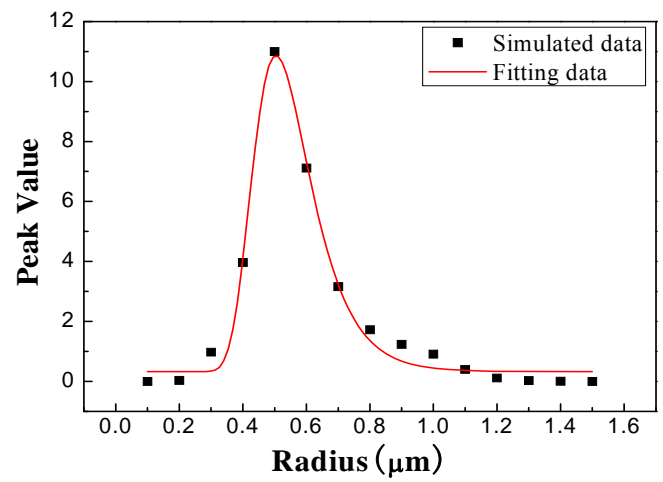

Figure 5. Different radiuses and its corresponding enhanced peak value for a metallic grating with the period of 2.9 micron and the enhanced wavelength is $4.5 \mu \mathrm{m}$.

erate optimized enhancement through surface plasmon. It is not difficult to derive the optimized radius of 0.5046 $\mu \mathrm{m}$ after fitting the data. However, it is apparent that the two values are close. The difference of several nanometers can be ignored for an array with dimension of several microns. Thus, the fitting accuracy is high enough for the curve of Figure 4. Hence, Equation (3) can be an empirical formula and provides a rough guide during the designing of the metallic grating for infrared band at least.

\section{Summary}

To investigate basic rules regarding metallic gratings being used for QWIP, we carried out optimization of duty ratio of the metallic gratings. For the periodic array with a certain period, we performed an investigation regarding changing trend of enhanced resonant peak and the duty ratio for different periods in infrared waveband, and analyzed the relationship between duty ratio and enhanced peak intensity. In addition, the curves of enhanced wavelength and duty ratio were also studied which can be used as a basic rule of the metallic grating. To prove validity of the trend and rule, we simulated a group of models with different radius which mean different duty ratios also. After comparison, the results demonstrated that the trend and rule are valid, and can be treated as a guide for further study of metallic grating in infrared band.

\section{Acknowledgements}

The research work was supported by National Natural Science Foundation of China (No. 60877021 and 61077010).

\section{References}

[1] T. W. Ebbesen, H. J. Lezec, H. F. Ghaemi, T. Thio and P. 
A. Wolf, "Extraordinary Optical Transmission through Sub-Wavelength Hole Arrays," Nature, Vol. 391, No. 6668, 1998, pp. 667-669.

[2] W. L. Barnes, A. Dereux and T. W. Ebbesen, "Surface Plasmon Subwavelength Optics," Nature, Vol. 424, No. 14, 2003, pp. 824-829.

[3] U. Schroter and D. Heitmann, "Surface-Plasmon-EnhanCed Transmission through Metalliclic Gratings," Physical Review B, Vol. 58, No. 23, 1998, pp. 419-421.

[4] E. Popov, M. Nevière, S. Enoch and R. Reinisch, "Theory of Light Transmission through Subwavelength Periodic Hole Arrays," Physical Review B, Vol. 62, No. 23, 2000, pp. 16100-16108. doi:10.1103/PhysRevB.62.16100

[5] A. Krishnan, T. Thio, T. J. Kim, H. J. Lezec, T. W. Ebbesen, P. A. Wolff, J. Pendry, L. Martin-Moreno and Garcia-Vidal, "Evanescently Coupled Resonance in Surface Plasmon Enhanced Transmission," Optics Communications, Vol. 200, No. 1-6, 2001, pp. 1-7.

[6] S. A. Maier, "Plasmonics: Fundamentals and Aplications," Springer, Science+Business Media LLC, 2007.

[7] H. Raether, "Surface Plasmons on Smooth and Rough Surfaces and on Gratings," Springer Tracts in Modem Physics, Springer-Verlag, Berlin, Heidelberg, Vol. 2, p. 1, 1988.

[8] H. Schneider and H. C. Liu, "Quantum Well Infrared Photodetectors,"Springer-Verlag, Berlin Heidelberg, 2007.

[9] Z.-B. Li, Y.-H. Yang, X.-T. Kong, W.-Y. Zhou and J.-G. Tian, "Enhanced Transmission through a Subwavelength Slit Surrounded by Periodic Dielectric Bars above the Metallic Surface," Applied Optics, Vol. 10, 2008, Article ID: 095202.

[10] H. P. Paudel, K. Bayat, M. F. Baroughi, S. May and D. W. Galipeau, "Geometry Dependence of Field Enhancement in 2D Metalliclic Photonic Crystals," Optics Express, Vol. 17, No. 24, 2009, pp. 22179-22189. doi:10.1364/OE.17.022179

[11] J. W. Cleary, G. Medhi, R. E. Peale and W. R. Buchwald, "Long-Wave Infrared Surface Plasmon Grating Coupler," Applied Optics, Vol. 49, No. 16, 2010, pp. 3102-3109. doi:10.1364/AO.49.003102

[12] W. L. Barnes, W. A. Murray, J. Dintinger, E. Devaux and T.W. Ebbesen, "Surface Plasmon Polaritons and Their Role in the Enhanced Transmission of Light through Periodic Arrays of Subwavelength Holes in a Metallic Film," Physical Review Letters, Vol. 10, No. 92, 2004, Article ID: 107401.

[13] A. Benabbas, V. Halté and J.-Y. Bigot, "Analytical Model of the Optical Response of Periodically Structured Metalliclic Films," Optics Express, Vol. 22, 2005, pp. 8730-8745. doi:10.1364/OPEX.13.008730

[14] L. Martin-Moreno and F. J. Garcia-Vidal, "Optical
Transmission Through Circular Hole Arrays in Optically Thick Metallic Films," Optics Express, Vol. 12, No. 16, 2004, pp. 3619-3628. doi:10.1364/OPEX.12.003619

[15] Y. Xie, A. R. Zakharian, J. V. Moloney and M. Mansuripur, "Transmission of Light through Periodic Arrays of Sub-Wavelength Slits in Metalliclic Hosts," Optics Express, Vol. 14, No. 14, 2006, pp. 6400-6413. doi:10.1364/OE.14.006400

[16] Lumerical Solution Inc., "FDTD Solution, A Commercial Professional Software," Lumerical Solution Inc., Vancouver, Canada. http://www.lumerical.com

[17] L. Salomon, F. Grillot, A. V. Zayats and F. de Fornel, "Near-Field Distribution of Optical Transmission of Periodic Subwavelength Holes in a Metallic Film," Physical Review Letters, Vol. 86, 2001, pp. 1110-1113. doi:10.1103/PhysRevLett.86.1110

[18] H. F. Ghaemi, T. Thio, D. E. Grupp, T. W. Ebbesen and H. J. Lezec, "Surface Plasmons Enhance Optical Transmission through Subwavelength Holes," Physical Review B, Vol. 58, No. 11, 1998, pp. 6779-6782. doi:10.1103/PhysRevB.58.6779

[19] J. G. Rivas, C. Schotsch, P. H. Bolivar and H. Kurz, "Enhanced Transmission of Thz Radiation through Subwavelength Hole," Physical Review B, Vol. 68, No. 20, 2003, Article ID: 201306.

[20] A. P. Hibbins, J. R. Sambles and C. R. Lawrence, "Gratingless Enhanced Microwave Transmission through a Subwavelength Aperture in a Thick Metallic Plate," Applied Physics Letters, Vol. 84, No. 24, 2002, pp. 46614663.

[21] M. Sarrazin, J.-P. Vigneron and J.-M. Vigoureux, "Role of Wood Anomalies in Optical Properties of Thin Metalliclic Films with a Bidimensional Array of Subwavelength Holes," Physical Review B, Vol. 67, 2003, Article ID: 085415. doi:10.1103/PhysRevB.67.085415

[22] K. J. K. Koerkamp, S. Enoch, F. B. Segerink, N. F. van Hulst and L. Kuipers, "Strong Influence of Hole Shape on Extraordinary Transmission through Periodic Arrays of Subwavelength Holes," Physical Review Letters, Vol. 92, 2004, Article ID: 183901. doi:10.1103/PhysRevLett.92.183901

[23] A. Degiron and T. W. Ebbesen, "The Role of Localized Surface Plasmon Modes in the Enhanced Transmission of Periodic Subwavelength Apertures," Journal of Optics A: Pure and Applied Optics, Vol. 7, No. 2, 2005, pp. S90S95. doi:10.1088/1464-4258/7/2/012

[24] L. Martin-Moreno, F. J. Garcia-Vidal, H. J. Lezec, K. M. Pellerin, T. Thio, J. B. Pendry and T. W. Ebbesen, "Theory of Extraordinary Optical Transmission through Subwavelength Hole Arrays," Physical Review Letters, Vol. 86, No. 6, 2001, pp. 1114-1117. doi:10.1103/PhysRevLett.86.1114 